\title{
DETERMINING AUTOMORPHISMS OF THE RECURSIVELY ENUMERABLE SETS ${ }^{1}$
}

\author{
RICHARD A. SHORE
}

\begin{abstract}
We answer two questions of A. Nerode and give information about how the structure of $\mathcal{E}^{*}$, the lattice of r.e. sets modulo finite sets, is determined by various subclasses.

THEOREM. If $e^{*}$ is any nontrivial recursively invariant subclass of $\mathcal{E}^{*}$, then any automorphism of $\mathcal{E}^{*}$ is determined uniquely by its action on $\mathcal{C}^{*}$.

THEOREM. If $e^{*}$ is the class of recursive sets modulo finite sets or $\mathfrak{R}^{*} \subseteq$ $e^{*} \subseteq \delta^{*}\left(\mathscr{R}^{*}=\right.$ maximal sets, $\delta^{*}=$ simple sets $)$ then there is an automorphism of (the lattice generated by) $\mathcal{C}^{*}$ which does not extend to one of $\mathcal{E}^{*}$.
\end{abstract}

One of the major areas of concern in recursion theory has traditionally been the structure of recursively enumerable sets as a lattice, $\mathcal{E}$, and, more particularly, that of $\mathscr{E}^{*}$, the lattice modulo finite sets. Formally this point of view was introduced in Myhill [1956], but much of the earlier work on r.e. sets can now be viewed in this light as well. Much important work in this area has been done by exploiting and analyzing a common algebraic tool: the group of automorphisms of the lattice. A general introduction to the study of $\mathcal{E}^{*}$ can be found in Chapter 12 of Rogers [1967]. For a short survey of more recent work as well as a major new result the reader should see Soare [1974] and [1974a].

In this paper we are concerned with the general question of how various subclasses such as $\Omega^{*}$, the recursive sets modulo finite sets, sit inside of $\mathcal{E}^{*}$ and to what extent they determine its structure at least as far as automorphisms are concerned. More specifically we begin with two questions of A. Nerode:

(1) Is every automorphism of $\mathcal{E}^{*}$ uniquely determined by its action on $\Re *$ ?

(2) Does every automorphism of $\Re *$ extend to one of $\mathcal{E}^{*}$ ?

Of course one can ask the same questions for other reasonable subclasses of $\mathcal{E}^{*}$ as well.

Upon first consideration it seemed that the answer to the first question should be no. Thus, for example, consider an $r$-maximal set $A$ (no recursive

Presented to the Society, August 27, 1976 under the title Determining automorphisms of $\mathcal{E}^{*}$; received by the editors September 13, 1976.

AMS (MOS) subject classifications (1970). Primary 02F25; Secondary 02F30.

Key words and phrases. Recursively enumerable sets, recursive sets, automorphisms of r.e. sets .

${ }^{1}$ The preparation of this paper was partially supported by NSF Grant MCS74-06378. We would also like to thank C. Jockusch, A. Nerode and R. Soare for valuable comments and suggestions. 
set splits $\bar{A})$ and a major subset $B$ of $A\left(A \cup W={ }^{*} N \Rightarrow B \cup W={ }^{*} N\right)$. Then $A$ and $B$ sit the same way with respect to all recursive sets. Indeed one can see that the lattices generated by $\Re^{*} \cup\{A\}$ and $\Re^{*} \cup\{B\}$ are isomorphic via a map which is the identity on $\Re^{*}$ and takes $A$ to $B$. One might then think that one could extend this to an automorphism on all of $\mathcal{E}^{*}$. This, however, is not the case. Indeed we give a positive answer to the first question in the most general setting in Theorem 5: Every automorphism of $\mathcal{E}^{*}$ is uniquely determined by its action on any recursively invariant class.

As one should now expect the answer to the second question is no. Indeed $r$-maximal sets are used below to provide a counterexample (Theorem 8$)^{2}$. Although a result as general as that of Theorem 1 seems harder to formulate, we supply examples of nonextendible automorphisms for most classes of interest (Theorem 10).

In this paper all named sets will be assumed to be r.e. We use "*" to mean "modulo finite sets". Thus " $A \subseteq \subseteq^{*} B$ " means that there are only finitely many elements in $A$ not in $B . N$ is the set of nonnegative integers. $\bar{A}$ is the complement of $A . f[A]$ is the range of $f$ restricted to $A$. We refer the reader to Rogers [1967] for all unexplained notation and terminology.

1. We work up to the final theorem by stages showing that automorphisms are determined by their action on various classes. Thus throughout this section $\varphi_{1}$ and $\varphi_{2}$ will be automorphisms of $\mathcal{E}^{*}$ which we will assume agree on some class and then try to show that $\varphi_{1}=\varphi_{2}$. In addition to giving different pictures of the structure of $\mathcal{E}^{*}$ some of the intermediate results will have other applications as well. We begin with the low sets. ( $A$ is low iff $A^{\prime} \equiv{ }_{T} \varnothing^{\prime}$.)

LEMMA 1. If $\varphi_{1}$ and $\varphi_{2}$ agree on the low sets, $\varphi_{1}=\varphi_{2}$.

Proof. Consider any set $A$. By Sacks [1966, Chapter 6, Corollary 1] or by an easy direct construction there are low sets $B$ and $C$ such that $A=B \cup C$. As $\varphi_{1}(B)={ }^{*} \varphi_{2}(B)$ and $\varphi_{1}(C)={ }^{*} \varphi_{2}(C)$ by assumption, $\varphi_{1}(A)={ }^{*} \varphi_{2}(A)$.

The next class we want is $\mathfrak{R}^{*}$, the class of all maximal sets (modulo finite ones). But we first need another fact.

Lemma 2. If $A$ is low (or even low ${ }_{2}$, i.e. $A^{\prime \prime} \equiv_{T} \varnothing^{\prime \prime}$ ) and $B-A$ is infinite then there is a maximal set $M$ with $\bar{M} \subseteq B-A$.

Proof. Let $f$ be a recursive function enumerating $B \cup A \cdot f^{-1}[A]$ is clearly Turing reducible to $A$ and so low . By Lachlan [1968a] there is a maximal set $M_{1} \supseteq f^{-1}[A] . f\left[M_{1}\right]$ is easily seen to be maximal in $B \cup A$. Thus by Lachlan [1968] there is a recursive set $R \supseteq \overline{B \cup A}$ with $(B \cup A)-f\left[M_{1}\right] \subseteq \bar{R} . R \cup$

\footnotetext{
${ }^{2}$ This result was proved jointly with $\mathbf{R}$. Soare and is included here with his permission.
} 
$f\left[M_{1}\right]$ is then the required maximal set $M$.

We note that this fact can be combined with a proof from Shoenfield [1976] to characterize the degrees of sets with exactly $n$ maximal (or hyperhypersimple) supersets.

Corollary. A degree a contains a set $A$ with exactly $n$ maximal (or hhs)

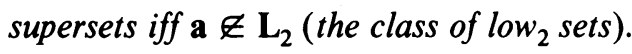

Proof. If $A$ is low $_{2}$ then (as it is not hyperhypersimple (hhs) by Martin [1966]) there are $B_{0}, B_{1}, \ldots$ such that the $B_{i}-A$ are infinite and pairwise disjoint. Thus by Lemma $2 A$ has infinitely many maximal supersets. Conversely if $\mathbf{a} \notin \mathbf{L}_{2}$ choose any set $B$ (with recursive enumeration $b(s)$ ) with exactly $n$ maximal (hhs) supersets. Now let $C$ be any set in a with recursive enumeration $c(s)$. If $A$ is the subset of $B$ permitted by $C$,

$$
A=\{x \mid(\exists t)(\exists s \leqslant t)(x=b(s) \text { and } c(t) \leqslant x)\}
$$

then Shoenfield's argument shows that $A$ also has exactly $n$-maximal (hhs) supersets. Of course $A \leqslant T C$ and by an unpublished remark of Jockusch one can choose $b$ and $c$ so that $B-A$ is infinite so that $A \equiv_{T} C$ : By Robinson [1968] we can choose $C$ and a recursive function $f$ so that $C_{c}$ (the computation function for $c$ ) does not dominate $f$. It is now easy to choose $b$ so that $C_{b}$ dominates $f$. By definition then $B-A$ is infinite.

Returning to our immediate concerns we prove

LEMMA 3. If $\varphi_{1}, \varphi_{2}$ agree on $\mathfrak{T} *$ then $\varphi_{1}=\varphi_{2}$.

Proof. If not, there is, by Lemma 1 , a low set $A$ with $\varphi_{1}(A) \neq^{*} \varphi_{2}(A)$. Without loss of generality assume that $\varphi_{1}(A)-\varphi_{2}(A)$ is infinite. By Lemma 2 and the fact that $\varphi_{2}$ is an automorphism (and so preserves the following property of $A: \forall B\left[B \complement^{*} A \Rightarrow(\exists\right.$ maximal $M)\left(A \subseteq^{*} M\right.$ and $\left.\left.\left.B \ell^{*} M\right)\right]\right)$ there is a maximal set $M \subseteq^{*} \varphi_{1}(A)-\varphi_{2}(A)$. So $\varphi_{2}^{-1}(M)^{*} \supseteq A$ and $\varphi_{1}^{-1}(M)^{*} \supseteq A$. As automorphisms map only maximal sets to maximal sets and $\varphi_{1}$ and $\varphi_{2}$ agree on $\Re^{*}$, so do $\varphi_{1}^{-1}$ and $\varphi_{2}^{-1}$. Thus $\varphi_{2}^{-1}(M)=\varphi_{1}^{-1}(M)$ for a contradiction.

Note that in this proof we only needed that $\varphi_{1}$ and $\varphi_{2}$ were elementary maps agreeing on $\Re^{*}$ and containing $\Re^{*}$ in their range. We will need this fact in the next section.

We now give the answer to the first question.

LEMMA 4. If $\varphi_{1}, \varphi_{2}$ agree on $\Re^{*}, \varphi_{1}=\varphi_{2}$.

Proof. If not there is, by Lemma 3, a maximal set $M$ with $\varphi_{1}(M)$ $\neq^{*} \varphi_{2}(M)$. As $\varphi_{1}(M)$ and $\varphi_{2}(M)$ are maximal $\varphi_{1}(M) \cup \varphi_{2}(M)={ }^{*} N$. We can therefore reduce these sets to get a recursive set $R$ with $R \subseteq \varphi_{1}(M)$ and $R \supseteq \overline{\varphi_{2}(M)}$ (so $R \underline{Z}^{*} \varphi_{2}(M)$ ). As before this is a contradiction since automorphisms map the recursive sets onto recursive sets.

Finally we prove the general result. 
TheOREM 5. Let $\mathcal{C}^{*}$ be any nontrivial (i.e. none of $\varnothing,\{\varnothing\},\{N\}$ ) class of r.e. sets (modulo finite sets) closed under recursive isomorphism. If $\varphi_{1}$ and $\varphi_{2}$ agree on $\mathcal{C}^{*}$ then $\varphi_{1}=\varphi_{2}$.

Proof. If not Lemma 4 tells us that there is a recursive $R$ such that $\varphi_{1}(R) \neq \varphi_{2}(R)$. Without loss of generality assume that $\varphi_{1}(R)-\varphi_{2}(R)$ is infinite. So then is $\varphi_{2}^{-1} \varphi_{1}(R)-R$. (It is, of course, recursive.) Let $W^{\prime} \in C^{*}$ ( $W^{\prime} \neq \varnothing$ or $N$ ). We can clearly find a $W$ recursively isomorphic to $W^{\prime}$ (and so in $\bigodot^{*}$ ) with $\bar{W} \subseteq \varphi_{2}^{-1} \varphi_{1}(R)-R$. (Just map $\overline{\varphi_{2}^{-1} \varphi_{1}(R)-R}$ to a recursive subset of $W$.) Thus $W \cup \varphi_{2}^{-1} \varphi_{1}(R)={ }^{*} N$ but $W \cup R \neq *^{*} N$. By assumption $\varphi_{1}^{-1} \varphi_{2}$ is the identity on $C^{*}$. Applying this to the first fact gives $W \cup R={ }^{*} N$ which contradicts the second.

As one can argue that any class of r.e. sets of recursion theoretic interest should be closed under recursive isomorphism, this theorem gives a positive answer to question one for all classes of interest.

2. In trying to find an automorphism of $\Re^{*}$ not extending to one of $\mathcal{E}^{*}$, we (R.I. Soare and the author) began with the observation that the extension lemma of Soare [1974] constructs automorphisms of $\Omega^{*}$ rather than $\mathcal{E}^{*}$ if one begins with $r$-maximal sets rather than maximal sets. More specifically, if $A$ and $B$ are $r$-maximal it constructs a permutation $p$ of $N$ such that $p$ induces an automorphism of $\Re^{*}$ and $p[A]=B$. The next step was to show that at least some $r$-maximal sets are distinguished by their relationship with the recursive sets. The grossest and most familiar distinction between $r$-maximal sets is whether or not they have a maximal superset. This property proved to be dependent on the relationship of the set to the recursive sets and so supplied the solution. We begin with that result.

LEMMA 6. Let $A$ and $B$ be r-maximal. If for every recursive set $R, A \cup R$ $=^{*} N \Leftrightarrow B \cup R={ }^{*} N$, then $A$ has a maximal superset iff $B$ does.

Proof. Assume the condition is satisfied and that $B$ has a maximal superset $M$. By maximality either $\bar{M} \subseteq{ }^{*} \bar{A}$ or $\bar{M} \subseteq{ }^{*} A$. In the first case $A \subseteq \subseteq^{*} M$ and we are done. In the second case $A \cup M={ }^{*} N$ and we can reduce $A$ and $M$ to get a recursive set $R$ such that $A \cup R=N$ and $R \subseteq M$. But as $B \subseteq M$ we have $B \cup R \subseteq M \neq^{*} N$ for a contradiction.

LEMMA 7. If $A$ and $B$ are $r$-maximal there is an automorphism $\varphi$ of $R *$ such that for every recursive $R, A \cup R={ }^{*} N \Leftrightarrow B \cup \varphi(R)={ }^{*} N$.

Proof. As we have mentioned, this is a consequence of the extension lemma of Soare [1974]. But rather than using the proverbial canon we give a direct construction. We will build two lists, $A, R_{0}, R_{1}, \ldots$ and $B, \hat{R}_{0}, \hat{R}_{1}, \ldots$, such that $\left\{R_{i} \mid i<\omega\right\}$ and $\left\{\hat{R}_{i} \mid i<\omega\right\}$ are lists of the recursive sets. We wish to guarantee that for any sequence $i_{1}, i_{2}, \ldots, i_{n}$ that $A \cap R_{i_{1}}$ $\cap \cdots \cap R_{i_{n}}$ is infinite iff $B \cap \hat{R}_{i_{1}} \cap \cdots \cap \hat{R}_{i_{n}}$ is infinite. By the easy proof of Theorem 1.3 of Soare [1974] this suffices to construct a permutation $p$ of $N$ 
such that $p[A]=B$ and $p\left[R_{i}\right]=\hat{R}_{i}$. This $p$ will therefore induce the desired automorphism of $\Re^{*}$.

We proceed by induction with a back and forth argument. Say we wish to add $R_{n+1}$ to the first list. The $R_{1}, \ldots, R_{n}\left(\hat{R}_{n}, \ldots, \hat{R}_{n}\right)$ have split $A(B)$ and $\bar{A}(\bar{B})$ into $2^{n}$ pieces in the obvious way. We are assuming by induction that for each such piece $S_{j}\left(j<2^{n}\right) S_{j} \cap A$ and $S_{j} \cap \bar{A}$ are infinite iff the corresponding $\hat{S}_{j} \cap B$ and $\hat{S}_{j} \cap \bar{B}$ are. We wish to construct $\hat{R}_{n+1}$ so that, for each $j<2^{n}, \hat{R}_{n+1}$ acts the same way on $\hat{S}_{j}$ as $R_{n+1}$ does on $S_{j}:$ If $S_{j} \cap R_{n+1}$ ${ }^{*} S_{j}$ let $\hat{T}_{j}=\hat{S}_{j}$. If $S_{j} \cap R_{n}={ }^{*} \varnothing$ let $\hat{T}_{j}=\varnothing$. Otherwise both $S_{j} \cap R_{n+1}$ and $S_{j} \cap \vec{R}_{n+1}$ are infinite. By $r$-maximality of $A$ either $S_{j} \cap R_{n+1}{ }^{*} \supseteq \vec{A}^{n+1}$ or $\varnothing \subsetneq^{*} S_{j} \cap R_{n+1} \subseteq \subseteq^{*} A$. In either case the corresponding relation holds for $\hat{S}_{j}$ and $B$. We can therefore choose a recursive $\hat{R} \subseteq \hat{S}_{j} \cap B$. We let $\hat{T}_{j}=\hat{S}_{j}-$ $\hat{R}$. Thus in each case $\hat{T}_{j}$ has the same relation to $\hat{S}_{j}$ as $R_{n+1}$ does to $S_{j}$. We let $\hat{R}_{n+1}=\bigcup_{j<2^{n}} \hat{T}_{j}$.

We can now combine these results to produce our nonextendible automorphism of $\Re^{*}$.

THEOREM 8. There is an automorphism $\varphi$ of $R *$ which does not extend to one of $\mathcal{E}^{*}$.

Proof. Let $A$ be an $r$-maximal set with a maximal superset and $B$ one without any (one exists by Lachlan [1968]). Let $\varphi$ be the automorphism of Lemma 7. If there were an extension $\psi$ of $\varphi$ to $\mathcal{E}^{*}$ then we would have for every recursive set $R$ that

$$
\begin{aligned}
\psi(A) \cup \psi(R) & ={ }^{*} N \Leftrightarrow A \cup R={ }^{*} N \Leftrightarrow B \cup \varphi(R) \\
& ={ }^{*} N \Leftrightarrow B \cup \psi(R)={ }^{*} N .
\end{aligned}
$$

As $B$ has no maximal superset Lemma 6 says that $\psi(A)$ has none either for our contradiction.

Although there does not seem to be an obvious best theorem along these lines for arbitrary classes we can handle the standard ones of interest in recursion theory by another type of argument. We will consider subclasses $C^{*}$ of $\mathcal{E}^{*}$ containing $\Re^{*}$ (the maximal sets) and contained in $\delta^{*}$ (the simple sets). What we will actually build is a proper elementary map $\varphi: \mathcal{E}^{*} \rightarrow \mathcal{E}^{*}$ mapping $C^{*}$ onto $C^{*}$. That $\varphi$ restricted to $C^{*}$ has no extension to an automorphism of $\mathcal{E}^{*}$ will then follow from the remarks to Lemma 3.

THEOREM 9. There is a proper elementary embedding $\varphi: \mathcal{E}^{*} \rightarrow \mathcal{E}^{*}$ with $\delta^{*} \subseteq \operatorname{range}(\varphi)$.

Proof. The construction is patterned on one used by Lachlan to show that there are $2^{\kappa_{0}}$ automorphisms of $\mathcal{E}^{*}$ (see Soare [1974, Theorem 1.1]). We first choose a strictly decreasing sequence $\left\{R_{i} \mid i<\omega\right\}$ of infinite recursive sets with $R_{0}=N$ such that for every $n, W_{n}{ }^{*} \supseteq R_{n+1}$ or $W_{n} \subseteq \subseteq^{*} \bar{R}_{n+1}\left(\left\{W_{n} \mid n<\omega\right\}\right.$ is a listing of the r.e. sets.): If $W_{n+1} \underline{\mathcal{E}}^{*} \bar{R}_{n}$ then $W_{n+1} \cap R_{n}$ is infinite and we can choose $R_{n+1}$ to be a recursive subset of $W_{n+1} \cap R_{n}$ (coinfinite in $R_{n}$ ). 
Otherwise we can take any infinite recursive set coinfinite in $R_{n}$ as $R_{n+1}$. Next let $A$ be an infinite recursive subset of $\bar{R}_{1}$ coinfinite in $\bar{R}_{1}$. We will choose a pairwise disjoint sequence $\left\{A_{i} \mid i<\omega\right\}$ of infinite recursive sets such that $\cup A_{i}=A$ and if $W_{n}$ is simple $W_{n}{ }^{*} \supseteq \cup_{i>n} A_{i}$ : By simplicity $W_{n} \cap A-$ $\bigcup_{i<n} A_{i}$ is infinite and so has an infinite recursive subset $R$ coinfinite in it. We let $A_{n+1}=A-\cup_{i<n} A_{i}-R$. (So $A_{j} \subseteq R \subseteq W_{n}$ for every $j>n$.) We have the following picture in mind:

\begin{tabular}{|l|l|}
\hline \multicolumn{2}{|c|}{$R_{0}-R_{1}$} \\
\hline & \multicolumn{2}{|l|}{$R_{2}-R_{3}$} \\
\cline { 2 - 2 }$R_{1}-R_{2}$ & \multicolumn{2}{|l|}{} & $\ldots$ \\
\hline
\end{tabular}

\begin{tabular}{|c|c|c|c|c|}
\hline \multicolumn{5}{|c|}{$R_{0}-R_{1}-A$} \\
\hline$A_{0}$ & $A_{1}$ & $A_{2}$ & \multicolumn{2}{|c|}{$\cdots$} \\
\hline \multirow{2}{*}{\multicolumn{3}{|c|}{$R_{1}-R_{2}$}} & \multicolumn{2}{|c|}{$R_{2}-R_{3}$} \\
\hline & & & & $\ldots$ \\
\hline
\end{tabular}

We now choose any recursive one-one onto maps $p_{n}: R_{n}-R_{n+1} \rightarrow\left(R_{n}-\right.$ $\left.R_{n+1}\right) \cup A_{n}$. Together these define a permutation $p$ of $N$ by $p(x)=p_{n}(x)$ where $x \in R_{n}-R_{n+1}$.

Claim 1. $p$ induces an elementary embedding $\varphi: \mathcal{E}^{*} \rightarrow \mathcal{E}^{*}$.

Proof. In fact for every $n$ the sets $p\left[W_{0}\right], p\left[W_{1}\right], \ldots, p\left[W_{n}\right]$ are the images of $W_{0}, W_{1}, \ldots, W_{n}$ under a single recursive permutation $\hat{p}_{n}$ of $N: \hat{p}_{n}(x)=$ $p_{i}(x)$ if $x \in R_{i}-R_{i+1}, i \leqslant n$, and $\hat{p}_{n}(x)=q_{n}(x)$ if $x \in R_{n+1}$ where $q_{n}$ is any one-one recursive map of $R_{n+1}$ onto $R_{n+1} \cup \cup_{i>n} A_{i}=R_{n+1} \cup(A-$ $\left.\cup_{i<n} A_{i}\right)$. To see this consider a $W_{i}, i \leqslant n . \hat{p}_{n}=p$ on $R_{n+1}$ so if $W_{i} \subseteq{ }^{*} \bar{R}_{n+1}$ then there is no problem. Otherwise however $W_{i}{ }^{*} \supseteq R_{n+1}$ by construction so

$$
\begin{aligned}
p\left[W_{i}\right] & ={ }^{*} \hat{p}_{n}\left[W_{i} \cap \bar{R}_{n+1}\right] \cup p\left[W_{i} \cap R_{n+1}\right] \\
& ={ }^{*} \hat{p}_{n}\left[W_{i} \cap \bar{R}_{n+1}\right] \cup R_{n+1} \cup \bigcup_{i>n} A_{i} \\
& ={ }^{*} \hat{p}_{n}\left[W_{i} \cap \bar{R}_{n+1}\right] \cup \hat{p}_{n}\left[W_{i} \cap R_{n+1}\right]={ }^{*} \hat{p}_{n}\left[W_{i}\right]
\end{aligned}
$$

Thus $p\left[W_{0}\right], \ldots, p\left[W_{n}\right]$ are the images of $W_{0}, \ldots, W_{n}$ under the automorphism induced by $\hat{p}_{n}$. They therefore satisfy the same first order sentences and so $\varphi$ is an elementary embedding.

Claim 2. $A \notin$ range $\varphi$.

Proof. Consider any $W_{n}$. If $W_{n}{ }^{*} \supseteq R_{n+1}$ then $\varphi\left(W_{n}\right)^{*} \supseteq R_{n+1}$ so $\varphi\left(W_{n}\right)$ $\neq^{*} A$. If $W_{n} \subseteq{ }^{*} \overline{R_{n+1}}$ then $\varphi\left(W_{n}\right) \cap \cup_{i>n} A_{i}={ }^{*} \varnothing$ and so $\varphi\left(W_{n}\right) \neq{ }^{*} A$.

Claim 3. If $W_{n}$ is simple then $W_{n} \in$ range $\varphi$.

Proof. $W_{n} Z^{*} \overline{R_{n+1}}$ by simplicity so $W_{n}{ }^{*} \supseteq R_{n+1}$. Moreover $W_{n}{ }^{*} \supseteq$ $\cup_{i>n} A_{i}$ by the choice of the $A_{i}$. Now $W=W_{n} \cap\left(R_{n+1}-\bigcup_{i>n} A_{i}\right)$ is an r.e. subset of $\overline{R_{n+1}}-\cup_{i>n} A_{i}$ which is the range of $\hat{p}_{n}$. Thus there is an r.e. set 
$U \subseteq \overline{R_{n+1}}$ (the domain of $\hat{p}_{n}$ ) such that $p[U]=\hat{p}_{n}[U]=W$. We now claim that $\varphi\left(U \cup R_{n+1}\right)=W_{n}$ :

$$
\begin{aligned}
\varphi\left(U \cup R_{n+1}\right) & =p\left[U \cup R_{n+1}\right]=p[U] \cup p\left[R_{n+1}\right] \\
& =W_{n} \cap\left(\overline{R_{n+1}}-\bigcup_{i>n} A_{i}\right) \cup R_{n+1} \cup \bigcup_{i>n} A_{i}=W_{n} .
\end{aligned}
$$

Note that if $W$ is any nonsimple set we could arrange for $W$ to be omitted from the range of $\varphi$ by choosing $A \subseteq \bar{W}$ and $R_{1} \subseteq W$. For then $W_{n}{ }^{*} \supseteq$

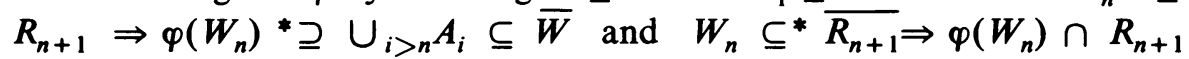
$={ }^{*} \varnothing$. So in either case $\varphi\left(W_{n}\right) \neq{ }^{*} W$. Thus, for example, we see that no nonsimple $W$ is algebraic (in the model theoretic sense) over $\mathcal{S}^{*}$. Again taking different choices for the $p_{n}$ we can get $2^{x_{0}}$ such elementary embeddings. We also get our main result on nonextendability as an application of this theorem.

THEOREM 10. Let $e^{*}$ be any subclass of $\mathcal{E}^{*}$ closed under recursive isomorphism such that $\mathfrak{K}^{*} \subseteq \mathcal{C}^{*} \subseteq \mathcal{S}^{*}$. Then there is an automorphism of the lattice $\mathcal{L}^{*}$ generated by $\mathcal{C}^{*}$ not extendible to one of $\mathcal{E}^{*}$.

Proof. We claim that the $\varphi$ of Theorem 9 restricted to $\mathcal{L}^{*}$ is the desired map.

Claim 1. $\varphi$ maps $\complement^{*}$ onto $C^{*}$ (and so restricts to an automorphism of $\complement^{*}$ ).

Proof. If $W_{n} \in \mathcal{C}^{*}$ then $\varphi\left(W_{n}\right)=\hat{p}_{n}\left[W_{n}\right]$ is recursively isomorphic to $W_{n}$ and so in $\mathcal{C}^{*}$. On the other hand if $W_{n} \in \mathcal{C}^{*}, W_{n} \in \mathcal{S}^{*}$ and so $W_{n}=\varphi(W)$ for some $W$. But as before $W \equiv W_{n}$ and so $W \in \mathrm{C}^{*}$.

Claim 2. No automorphism $\psi$ of $\mathcal{E}^{*}$ extends $\varphi$.

Proof. By the remarks to Lemma 3 as $\varphi$ and $\psi$ are elementary and agree on $M^{*} \subseteq e^{*}$ (which is also in their range) we would have to have $\varphi=\psi$. Of course, this contradicts the properness of $\varphi$.

We can also prove some additional results along these lines. Thus, for example, using the ideas from Shore [1977] and the full force of Theorem 5 we can remove the restriction that $\mathfrak{K}^{*} \subseteq \mathrm{C}^{*}$. We can also construct proper elementary embeddings containing $\Re^{*}$ in their range (and omitting any prescribed nonrecursive set). This, of course, gives an alternate proof of Theorem 9 by using Lemma 4 instead of Lemma 3. Indeed even some combinations of the two embedding constructions are possible. We do not, however, know what the most general theorem along these lines should be. Perhaps one could show that for any recursively invariant class $\mathcal{C}$ * not generating all of $\mathcal{E}^{*}$ there are proper elementary embeddings of the above sort containing $e^{*}$ in their range and so that there are nonextendible automorphisms of the lattice generated by $e^{*}$. In any case returning to our original question of how the structure of $\mathcal{E}^{*}$ is determined by subclasses the answer in general seems to be that no recursively invariant subclass (or at least none of the usual ones) determine $\mathcal{E}^{*}$ in a first order way but that all of 
them fix the structure of $\mathcal{E}^{*}$ in a second order way in terms of automorphisms.

\section{BIBLIOGRAPHY}

A. H. Lachlan [1968], On the lattice of recursively enumerable sets, Trans. Amer. Math. Soc. 130, 1-37.

[1968a], Degrees of recursively enumerable sets which have no maximal superset, J. Symbolic Logic 33, 431-443.

D. A. Martin [1966], Classes of recursively enumerable sets and degrees of unsolvability, Z. Math. Logik Grundlagen Math. 12, 295-310.

J. Myhill [1956], The lattice of recursively enumerable sets, J. Symbolic Logic 21, 220.

R. W. Robinson [1968], A dichotomy of the recursively enumerable sets, Z. Math. Logik Grundlagen Math. 14, 339-356.

H. Rogers, Jr. [1967], Theory of recursive functions and effective computability, McGraw-Hill, New York.

G. E. Sacks [1966], Degrees of unsolvability, 2nd. ed., Princeton Univ. Press, Princeton, N.J.

J. R. Shoenfield [1976], Degrees of classes of r.e. sets, J. Symbolic Logic 41, 695-696.

R. A. Shore [1977], Nowhere simple sets, J. Symbolic Logic (to appear).

R. I. Soare [1974], Automorphisms of the lattice of recursively enumerable sets. I: Maximal sets, Ann. of Math. (2) 100, 80-120. Soc. 80, 53-58.

[1974a], Automorphisms of the lattice of recursively enumerable sets, Bull. Amer. Math.

Department of Mathematics, University of Illinois at Chicago Circle, Chicago, ILLINOIS 60680

Department of Mathematics, Cornell University, Ithaca, New York 14853 (Current address) 